\title{
The Study of Visual Symbols in Digital Media Technology
}

\author{
Wang Xiaoyan, Zhao Hui \\ Shandong Xiehe University, Jinan, Shandong, China, 250109
}

Keywords: visual symbol; digital media technology; guide

\begin{abstract}
Visual symbols is a bridge of communication between the spiritual civilization and material civilization. The unique expression techniques and rich image features of visual symbols expand people's experience field and play an important role in people's grasp of the objective world knowledge. Digital media is also the earliest medium in the form of avant-garde art. Once the technology of digital media can be transformed into practical application by experiment, it can be popularized effectively. Digital media technology will be the new focus after the Internet. With the relative maturity of digital technology, visual symbols have been paid more attention in the field of visual communication. Thus, the research on visual symbol and information dissemination is carried out successively.
\end{abstract}

\section{Introduction}

The rapid development of the information age and have made great changes in our society. In the 20th century, visual information transmission has been stuck in two-dimensional space to deal with plane factors, including text, graphics, format, binding, etc. By the 1990s, digital media art was inseparable from our human activities and digital media became an indispensable part of human life. The change of digital media technology has many influences in various fields, and it is represented by two typical fields of art and media. In the field of art, digital media technology presents special inspirations, it has changed the traditional art creation techniques and injected the elements of science and technology. The emergence of digital media technology is also a model of stage development. With the development of information technology, especially the Internet which was born in combination with experiment. With the development of digital technology and media technology, new media emerged after the popularization of the Internet. New media gradually infiltrated into people's lives. The design and dissemination of information began as a form of visual communication.

\section{The Representation of Visual Symbols in Digital Media Technology}

Information transmission in various forms is developed with the rapid development of social economy. Digital media technology also adds a lot of bright spots to the whole media world, which bringing many opportunities. Through this virtual reality technology, digital media technology enables people to communicate with visual symbols, such as graphics, color, text, images, sound and animation. The range of digital media technology is the further development of human senses. At the same time, the expansion of visual communication is the meaning of dynamic visual symbol. As an interactive digital media technology, the Internet is a fairly rapid tool for absorbing new information at present, and it is also the first choice for communication methods. From the perspective of its structure, the network still brings together a lot of visual communication theories to transmit information symbols. In the field of information communication, visual art also has a new expression pattern. The reason why it can improve the speed, efficiency and scope of information and visual communication is that it is inseparable from its unique propagation characteristics. The spread of visual arts has little to do with economic, cultural and political obstacles. Visual symbol is a combination of applied communication and art. It is more dependent on audience to watch. Under the premise of the whole digital media, the audience becomes the consumer information. As for the party that disseminates information, it also starts to have 
information optional, which directly determines the aesthetic feeling of visual symbols, which we still need to extend.

\section{The Propagation Characteristics of Dynamic Visual Symbols}

\subsection{Immersive}

The first step in the combination of art may not be a product of practicality, but it has great potential to transform into applicability, and it's a predictor of the future of life. Digital media is like a collage artist who can improvise a work on a network. As a carrier of information, dynamic visual symbols play a multi-dimensional role in conveying information. As the director of this multi-dimensional role, the designer must look through the whole society and face the challenges brought by the development of science and technology.

\subsection{Linking}

The visual symbol in digital media is not only the function of visual transmission, a good UI design not only makes the software become personal and tasteful, but also the operation of the software becomes comfortable, simple and free. Which fully reflects the positioning and characteristics. In addition, it acts as a chain of information.

\subsection{Instant replication}

Digital media transmission of graphics, images, sounds and other information in the form of digital coding. The other end of the receiving message can be presented to the audience without loss. This is the most prominent feature of digital modern communication.

\subsection{Virtual}

The virtuality of digital art is reflected in speed. The network digital media represents the instantaneous dissemination of information, thus creating a new type of human interaction and interactio especially in the field of thought. In this space, the exchange of design information is not only convenient, but also becomes tangible and perceptible. Although this environment is more abstract, it does not become unreal in the representation, feeling and experience of design information.

\subsection{Interactivity}

The interactivity of visual symbols is one of the most typical expressions in digital media art. In all artistic forms, only digital expressive art can achieve interactivity. Audience is no longer a passive accept fixed in fixed time and space of art information, but can have a choice and more directly involved in the digital media information dissemination process. Digital technology integrates digital information processing technology, computer technology, digital communication technology and network technology to make abstract information that can sense management and interaction.

\subsection{The nonlinear}

In the artistic creation of digital media, the "non-linear" propagation of visual symbols is an expression of artistic language, which is the exploration of time reconstruction. Nonlinear design make visual symbol information can be arbitrary cut, and even in accordance with the design intent, present and future was completely broken.

\subsection{Diversity}

Pluralism refers to the unity of various forms of artistic expression, including sound, image, picture, animation and music. With the development of modern digital science and technology, the way of using words to transmit information has become increasingly unsuitable for the development of modern society. When consumers accept the art of digital media, the experience is full. Besides the presentation of pure text, there are also visual, auditory and even taste and touch. 


\subsection{The 3D sex}

The visual symbol becomes omnipotent as the carrier of digital technology. In today's real life, people are always cut off by time or space, and cannot reach a corner of the earth. Sometimes there are other restrictions, such as physical or economic factors. With digital technology, we can go to any place where we want to go, without having to travel in person, such as google map, street view and so on. Some of the software can even simulate visitors walking on the map in real time, or even the number of cars on the street.
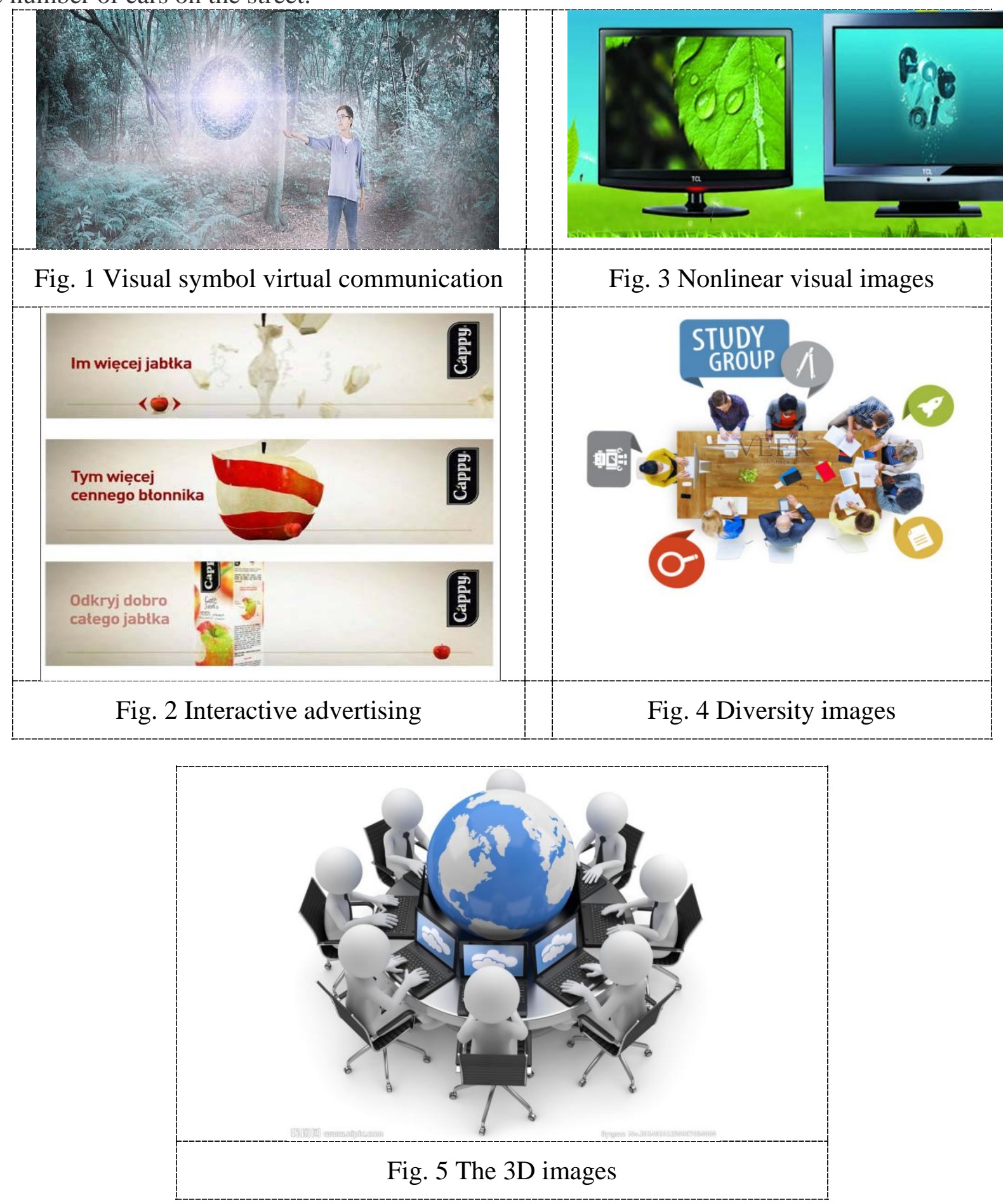

\section{The Orientation of Visual Symbols in Digital Media Technology}

\subsection{Promoting the development of digital media technology}

Digital visual design is characterized by immaterial, alternating and multidimensional language. The static effect of plane is moving towards dynamic and interactive audio-visual development. The 
single print media is developing towards the integration and spread of multi-media. Based on the change of design language and communication form, people have new cognition and aesthetic characteristics of visual communication design in digital media environment. And visual symbols are increasingly important. Dynamic visual communication has obvious advantages in information transmission compared with print design. The text in the dynamic visual communication is different from the text design in the printing design. It has the properties of time and space, motion and ideation. In dynamic visual communication, the text is mainly expressed through font features, font structure, graphical and auxiliary elements of fonts, etc. In the dynamic text design, we should adopt the principle of accurate transmission of information, and constantly create dynamic text design with diversified styles. In order to make the text convey information more effectively, the dynamic text is designed and applied to create a better information communication environment.

\subsection{The vision of visual communication design}

Visual communication design is not isolated, and its development is inseparable from the change of media environment. With the rapid development of digital media, the impact of new media on traditional visual communication design is inevitable. Facing the opportunities and challenges in the digital age, we should explore new design methods that meet the development needs based on the original graphic design thinking. The emergence of new design field does not mean the disappearance of the old field, but the constant innovation of visual communication design in the continuous learning and integration. In the era of digital media, the development of visual communication design increasingly reflects the dependence on technology, especially the dynamic development trend of visual communication design. We should make full use of the advanced technology to serve the effective expression design concept rather than the pure technology infatuation.

\subsection{Humanistic care of visual symbols}

In the age of digital information, information science and network has changed people's life style and attitude. For design, the focus is not only on basic functional satisfaction, but on the pursuit of emotional level. The human nature of visual symbol design realizes the emotional communication between people through the materialized works. Nowadays, with the rapid development of digital technology and virtual reality, the high technology of the design is amazing. At the same time, it also causes people to have psychological distance, so modern visual design is more and more concerned with people's emotional needs. In visual communication design in the process of digitalization, the integration of humanistic concept is very necessary, so the development of digital visual design need support of humanistic thought. Contemporary visual communication design is more about service to business and market, thus neglecting its value as a kind of cultural behavior

and aesthetic behavior. In the process of the development of visual communication design, we should focus on humanistic element, indigenous elements and the combination of digital technique, thus creating design work conforms to the national culture characteristics. In today's globalized world, regional culture is becoming less and less different, and what designers can do is to continuously strengthen national cultural identity through design.

\section{Conclusion}

Every era is the use of productivity to promote the development of art. Digital media technology has brought about great changes in visual arts, one of the most notable is the visual symbol in digital media technology. Visual symbol is along with the development of productivity, it forms of open, change quickly, language, which like the dynamic and engaging visual effect. But as a new form of art, it also has many disadvantages, which is limited to the most of the theoretical research. There is no practice in gymnastics and experience accumulation, and we will continue to focus on digital media technology in the future. 


\section{References}

[1] Li, Y. -Y. Research on ritual and performance of lion dance in Taiwan: The cases of Taiwanese Lion and Cantonese Lion; Taipei; National Taipei University Institute of Folk Art; [D]. National Taipei University Institute of Folk Arts Master Master's thesis. 83-87 (1992)

[2] Lewis, M and Jacobson, J. Games Engines in Scientific Research. Communications of ACM, 27-31 (2002)

[3] Macedo, L, Cardoso, A. Modelling Forms of Surprise in an Artificial Agent, in Proc. of the 23rd Annual Conference of the Cognitive Science Society. 23rd Annual Conference of the Cognitive Science Society, Edinburgh. 588-593 (2001)

[4] Moser, M. A. (Ed.), Immersed in Technology: Art and Virtual Environments. Cambridge (Massachussets), MIT Press, 96-88 (2012)

[5] Mateas, M., Stern, A. Interaction and Narrative. In: Zimmerman, E., Katie, S. (eds.) The Game Design Reader: A Rules of Play Anthology. The MIT Press, Cambridge. 642-669 (2006)

[6] Tanenbaum, J. Tomizu, A. Affective Interaction Design and Narrative Presentation. In: AAAI Fall Symposium on Intelligent Narrative Technologies. AAAI Press, Arlington. 88-90 (2007)

[7] Tanenbaum, J., Tanenbaum, K. The Reading Glove: designing interactions for object based tangible storytelling. In: International Conference on The Augmented Human. ACM Press, Mageve 65-68 (2010)

[8] Tanenbaum, K., Hatala, M., Tanenbaum, J. User Perceptions of Adaptivity in an Interactive Narrative. In: Konstan, J. A., Conejo, R., Marzo, J. L., Oliver, N. (eds.) UMAP. 124-128 (2011)

[9] Tanenbaum, K., Tanenbaum, J., Antle, A. N., Bizzocchi, J., Seif El-Nasr, M., Hatala, M.: Experiencing the Reading Glove. In: International Conference on Tangible and Embedded/ Embodied Interaction. ACM Press, Funchal. 231-235 (2011) 